\title{
NEONATAL DENERVATION INHIBITS THE NORMAL POSTNATAL DECREASE IN ENDPLATE CHANNEL OPEN TIME ${ }^{1}$
}

\author{
STEPHEN M. SCHUETZE*,2 AND STEFANO VICINI \\ * Department of Biological Sciences, Columbia University, New York, New York 10027 and $\ddagger$ Fidia Research Laboratories, \\ 35031 Abano Terme, Italy
}

Received November 29, 1983; Accepted February 24, 1984

\begin{abstract}
The apparent mean channel open time $(\tau)$ of acetylcholine receptors (AChR) at skeletal muscle endplates decreases $>3$-fold during development. In rat soleus muscles, the change occurs between postnatal days 8 and 18 as channels with long apparent open times $(\tau=4.5 \mathrm{msec})$ disappear while channels with short apparent open times $(\tau=1.5 \mathrm{msec})$ increase in number. We studied the role of innervation in this process by denervating neonatal soleus muscles prior to channel conversion. Tau at the denervated endplates was assayed at various times between days 8 and 18 by using fluctuation analysis. We found that early denervation blocked, or at least delayed, channel conversion. Unexpectedly, there was enhanced extrajunctional ACh sensitivity in the innervated muscles contralateral to the denervated ones. 'This observation allowed us to compare the apparent open times of junctional AChRs with those of extrajunctional AChRs $200 \mu \mathrm{m}$ distant in the same innervated fibers. In developing muscles, $\tau$ at the extrajunctional sites decreased in parallel with $\tau$ at the endplates. Thus, neural regulation of AChR channel gating extends well beyond the endplate boundaries.
\end{abstract}

Developmental interactions between nerve and muscle cells have been of great interest in recent years (for review see Dennis, 1981). Neuronal regulation of acetylcholine receptors (AChRs) has received special attention (Fambrough, 1979). During the formation of skeletal muscle endplates, nerve terminals influence the distribution (Anderson et al., 1977; Frank and Fischbach, 1979), the metabolic stability (Berg and Hall, 1975; Reiness and Weinberg, 1981), and the channel kinetics (Schuetze and Fischbach, 1978; Sakmann and Brenner, 1978; Fischbach and Schuetze, 1980) of underlying AChRs.

One striking change associated with innervation is a 3 -fold decrease in the apparent mean channel open time $(\tau)$ of AChRs (Schuetze and Fischbach, 1978; Sakmann and Brenner, 1978; Fischbach and Schuetze, 1980). Tau is about $4.5 \mathrm{msec}$ at $21^{\circ} \mathrm{C}$ in uninnervated, embryonic at myotubes (Fischbach and Schuetze, 1980), compared to about $1.5 \mathrm{msec}$ in mature endplates (Sakmann and Brenner, 1978; Fischbach and Schuetze, 1980 ). In rat soleus muscle endplates, the decrease in mean open time occurs between postnatal days 8 and 18 as channels with long apparent open times (slow channels) gradually disappear while channels with short apparent open times (fast channels) increase in number (Sakmann and Brenner, 1978).

The importance of the nerve for channel conversion is indicated by studies of AChRs in denervated muscles (Katz and Miledi, 1972). In denervated adult rat muscles, the newly syn-

\footnotetext{
${ }^{1}$ We thank Drs. R. Pollock, L. Rubin, and S. Siegelbaum for helpful discussions and Ms. B. Moss for technical assistance. This work was supported by grants from the National Institutes of Health (NS17774), the Muscular Dystrophy Association, and the March of Dimes. S. M. S. is a Sloan Research Fellow.

${ }^{2}$ To whom correspondence should be addressed.
}

thesized AChRs that appear in extrajunctional membrane regions have the long apparent open times characteristic of immature muscle fibers (Sakmann, 1978; Fischbach and Schuetze, 1980). In contrast, many of the AChRs in the endplate region have short apparent open times even several weeks after denervation, when presumably nearly all of the AChRs present at the time of denervation have been replaced (Brenner and Sakmann, 1983).

To explain these data, it has been proposed that the nerve mediates channel conversion not by altering or replacing the AChRs themselves, but by changing the membrane environment of endplate channels (Brenner and Sakmann, 1983). According to this idea, during postnatal development the endplate membrane is modified in some way so as to favor short gating times. One possibility is that the formation of postsynaptic folds, which occurs at the same time as channel conversion (Korneliussen and Jansen, 1976; Rosenthal and Taraskevich, 1977), may be related to the developmental $\tau$ decrease. A corollary of this hypothesis is that channel conversion is restricted to the endplate region.

This argument is supported by the finding that in adult human intercostal muscle, $\tau$ is short at the endplate but long at the ACh-sensitive (but aneural) myotendinous junction (Cull-Candy et al., 1982). However, other data indicate that channel conversion extends well beyond the endplate. In Xenopus myotomal muscle, the developmental decrease in $\tau$ occurs both at endplates and in extrajunctional regions (Kullberg et al., 1981). These apparently conflicting results may indicate a species difference.

We have tested the role of the nerve in regulating channel conversion in two ways. First, we followed the fate of AChRs in neonatal endplates that were denervated prior to channel 
conversion. We report that neonatal denervation arrests, or at least delays, the decrease in $\tau$. Second, we have studied the gating properties of $\mathrm{AChRs}$ in extrajunctional regions of developing fibers in innervated muscles. We report that channel conversion occurs not only at the endplate but also at extrajunctional sites up to at least $200 \mu \mathrm{m}$ (more than 10 endplate diameters) distant. Electron microscopy showed that these extrajunctional sites lacked any postsynaptic specializations. We conclude that channel conversion is independent of the formation of postsynaptic folds and of other developmental events unique to the endplate region. Although the mechanism underlying the $\tau$ decrease remains obscure, it is clear that its actions extend well beyond the immediate vicinity of motor nerve terminals.

\section{Materials and Methods}

Denervation. Neonatal Long-Evans rats (Pocono Rabbit Farms) were anesthetized with ether, and the lower left hindlimbs were denervated by removing a length of the sciatic nerve from the thigh. Six successful experiments were done using animals denervated between days 2 and 6 . One experiment was performed on an animal denervated on day 10.

Electrophysiology. Animals were decapitated, and one or both soleus muscles were removed. The muscles were pinned to a layer of cured Sylgard resin and bathed in well oxygenated L-15 tissue culture medium throughout the experiment. Under these conditions the muscles remained healthy for at least 8 to $10 \mathrm{hr}$. The experimental chamber was placed on the stage of a Zeiss UEM microscope equipped with differential interference contrast (Nomarski) optics. Using a $\times 40$ waterimmersion objective, we could clearly visualize the endplates of nor mally innervated muscle fibers. The bath temperature adjacent to the muscle was monitored constantly using a telethermometer (Yellow Springs Instrument Co.)

The apparent mean channel open time of AChRs was determined by fluctuation analysis of focal extracellular recordings of ACh-induced membrane currents (Katz and Miledi, 1972; Anderson and Stevens, 1973; Schuetze, 1980). At each test site, a pipette filled with $20 \mu \mathrm{M} \mathrm{ACh}$ was lowered onto the membrane. The pipette served both to deliver $\mathrm{ACh}$ and to record the induced currents. The pipettes were similar to those used for patch-clamping (Hamill et al., 1981) except that here the tips were somewhat larger ( 3 to $5 . \mu \mathrm{m}$ across). This technique also differed from patch-clamping in that there was no need to form a seal between the pipette tip and the membrane.

It should be noted that, although the ACh concentration within the pipette barrel was relatively high, it is likely that the actual concentration at the AChRs was several-fold lower. First, ACh in the tip of the pipette was undoubtedly diluted by the bath medium. Second, in recordings at endplate sites, $\mathrm{ACh}$ diffusing out of the pipette had to pass through the basal lamina and its associated AChE, which hydrolyzed some fraction of the transmitter before it could reach the synaptic AChRs. Previous experiments have shown that recordings made with this technique do not violate the "low ACh concentration limit" assumption of fluctuation analysis (cf. Anderson and Stevens, 1973) and give valid estimates of $\tau$ (Schuetze, 1980).

The current noise recordings were band-pass filtered (2 to $900 \mathrm{~Hz}$; Krohnhite) and fed directly into the analogue-to-digital converter of a Digital Equipment Corporation LSI 11/23 computer. Each spectrum was calculated on-line from 8096 digital samples obtained at $2 \mathrm{KHz}$. Care was taken to exclude segments of records that contained obvious artifacts or superimposed miniature endplate currents. A control spectrum, obtained with the pipette positioned away from the muscle fiber, was subtracted from each experimental spectrum to obtain a difference spectrum. Other details can be found in Schuetze (1980).

A simple Lorentzian curve was fitted to each difference spectrum by a nonlinear, weighted least-squares regression algorithm. When a test site contained a homogeneous population of AChRs, a good estimate of $\tau$ was derived from the critical frequency $\left(f_{c}\right)$ of the fitted curve through the relation $\tau=1 /\left(2 \pi f_{c}\right)$ (Anderson and Stevens, 1973). At many of the control, innervated endplates tested in this study (Tables I and II), the power spectra were clearly biphasic, indicating that they contained a mixture of channels with long and short apparent open times (Sakmann and Brenner, 1978; Fischbach and Schuetze, 1980). Although the spectra at these endplates are better fitted with the sum of two Lorentzians with different $f_{c}$ values, they were nevertheless, fitted with a single Lorentzian curve in order to save time during online analysis. In such cases, the apparent $\tau$ obtained from the single Lorentzian gives a rough estimate of the mean $\tau$ of all of the AChRs at the test site. This apparent $\tau$ decreases to the adult value $(\sim 1.5 \mathrm{msec})$ as the fraction of channels with short $\tau$ values increases, although the relationship is nonlinear.

$A C h E$ staining. Living soleus muscles were stained for $\mathrm{AChE}$ aclivity using the technique of Karnovsky and Roots (1964). Muscles were exposed to full-strength staining solution until patches of reaction product were visible (10 to $20 \mathrm{~min}$ ). 'The muscles then were washed in well oxygenated L-15 for $10 \mathrm{~min}$ before they were studied electrophysiologically.

Electron microscopy. In several experiments we examined the ultrastructure of cells that had been studied physiologically. Cells were labeled with a modification of a technique described by Maranto (1982) This technique is based on the observation that when Lucifer Yellow is excited with UV light, the fluorescing dye catalyzes the precipitation of diaminobenzidine (DAB) into an clectron-dense marker.

After a cell was assayed for mean channel open time, it was filled with Lucifer Yellow by iontophoresis $(-0.5$ to $-2 \mathrm{nA}$ DC for 2 to 5 $\mathrm{min}$ ) from an intracellular micropipette. The muscle was then fixed in $4 \%$ paraformaldehyde in $0.1 \mathrm{M}$ sodium cacodylate buffer $(\mathrm{pH} 7.4)$ for $20 \mathrm{~min}$ at room temperature, rinsed three times $(5 \mathrm{~min}$ each) in buffer, and incubated in $1 \mathrm{mg} / \mathrm{ml}$ of $\mathrm{DAB}$ in buffer. The preparation was put on the stage of the physiology microscope and examined with a Zeiss $\times 40$ water-immersion objective.

After relocating the Lucifer-filled cell, we epi-illuminated the fiber with UV light (Zeiss 100-W mercury lamp used with a fluorescein filter set) passed through the $\times 40$ objective. By stopping down the UV light source, we selectively illiminated precisely that portion of the cell that had been assayed for apparent mean channel open time. After about $15 \mathrm{~min}$, the illuminated part of the cell ( $\sim 10$ to $15 \mu \mathrm{m}$ across) turned dark brown. The muscle was then washed and fixed overnight at $4^{\circ} \mathrm{C}$ in $3 \%$ glutaraldehyde in buffer. The rest of the processing for electron microscopy was conventional.

\section{Results}

Tau at denervated endplates. To determine whether the normal $\tau$ decrease at endplates requires continued innervation, soleus muscles were denervated several days before the onset of channel conversion. Tau at these denervated endplates was assayed 2 to 14 days later.

We could not readily locate the former endplates in denervated muscles electrophysiologically. However, we could identify endplates histochemically by staining the living tissue for AChE activity. Since synaptic AChE remains highly localized following denervation (Miledi and Slater, 1968), the AChE reaction product marked the vacated endplate sites.

The overall staining pattern of denervated muscles resembled that of normal, innervated muscles. In both cases, each fiber contained a single patch of $\mathrm{AChE}$ reaction product. The distribution of patches was indistinguishable from the distribution of endplates in innervated muscles. Unlike $\mathrm{AChE}$ stain in muscle preserved with aldehydes, which is stable, AChE stain in living muscle faded and became undetectable 10 to $30 \mathrm{~min}$ after the preparation was washed. Physiological measurements were done only while the stain was visible.

The apparent mean channel open time of AChRs was measured by spectral analysis of focal extracellular recordings of ACh-induced membrane currents (Katz and Miledi, 1972; Anderson and Stevens, 1973; Schuetze, 1980). An ACh-filled pipette was positioned over each test site, and the resulting membrane currents were analyzed on-line with a digital computer. This technique has very good spatial resolution and assays essentially only those AChRs directly beneath the electrode tip (cf. Plate 1 in Schuetze, 1980). This is especially important in the present study because endplate AChRs in denervated muscles are surrounded by extrajunctional AChRs. The high resolution of extracellular recording minimizes the contamination of endplate recordings by currents flowing through nearby extrajunctional AChRs. 
We were unable to detect any ACh-induced currents at denervated endplates immediately after they were stained for AChE, presumably because the AChRs were desensitized by the high concentration of acetylthiocholine $(1.7 \mathrm{mM})$ in the staining solution. However, $\mathrm{ACh}$ sensitivity generally returned within several minutes, and measurements were made then. In some cases the recovery of ACh sensitivity coincided with the loss of visible AChE stain. When this happened, the preparation was either restained or discarded.

Staining the muscles for AChE had no major effect on $\tau$ (Table I). The mean $\tau$ measured at randomly selected extrajunctional sites on denervated muscles was the same before and after staining for AChE. Furthermore, the mean apparent open time at six innervated endplates was not changed significantly by staining. We also noted that AChE staining had no significant effect on the muscle resting potential, but it did abolish spontaneous transmitter release (data not shown). This latter phenomenon was not investigated further.

Spectra obtained from denervated and innervated endplates differed significantly. Figure 1 shows an example from two day18 fibers. The estimated $\tau$ values were $1.2 \mathrm{msec}$ at the innervated endplate and $4.2 \mathrm{msec}$ at the denervated endplate. Long apparent open times were also found at 27 other denervated endplates in 7 different soleus muscles (Table II). In every case, $\tau$ at the vacated endplate was longer than $\tau$ at innervated control endplates of the same age and similar to that found in uninnervated, embryonic muscle.

As shown in Table II, the mean $\tau$ at denervated endplates appeared to decrease with time, although the decrease was much smaller than at innervated endplates. This raises the possibility that denervation may delay the $\tau$ decrease rather than prevent it. Our data cannot distinguish between these hypotheses. Because of the small sample size, the differences in the mean $\tau$ values at denervated endplates of different ages are not statistically significant (Table II).

Moreover, the shortest $\tau$ estimates were recorded from two endplates in a day-18 muscle that was denervated at day 10 , or 4 days later than any of the others. Spectra at these endplates were biphasic, indicating that these cells did, in fact, have a subpopulation of adult-type fast channels. Since channel conversion in soleus typically begins at day 8 (Sakmann and Brenner, 1978), however, it is likely that the fast channels had appeared before denervation. We found no evidence for fast channels in any other denervated endplates. A study of day-18 muscles that were denervated at earlier times would be useful, but we found such muscles too atrophic for successful physiology experiments.

\section{TABLE I}

$\tau$ before and after AChE staining

Tau was estimated by fluctuation analysis before and after the muscle was stained for AChE activity. The extrajunctional sites tested before and after staining were not the same. The data were obtained from eight denervated muscles that were between 9 and 18 days old. In the case of the innervated endplates, the same six sites in one day-11 muscle were tested under the two conditions. The innervated endplates were located before staining by focal extracellular recording of spontaneous miniature endplate currents (Fiscibach and Schuetze, 1980; Schuetze, 1980). After staining, the AChE reaction product on the six test fibers was localized to the physiologically identified sites, as expected. All data were normalized to $21^{\circ} \mathrm{C}$ using a standard curve that was determined experimentally (Fischbach and Schuetze, 1980).

\begin{tabular}{lll}
\hline & \multicolumn{2}{c}{$\tau$} \\
\cline { 2 - 3 } & \multicolumn{1}{c}{ Before Stain } & \multicolumn{1}{c}{ After Stain } \\
\hline & \multicolumn{2}{c}{ mean \pm SEM, msec } \\
Extrajunctional sites & $4.33 \pm 0.15(n=39)$ & $4.36 \pm 0.15(n=13)$ \\
Innervated endplates & $3.33 \pm 0.19(n=6)$ & $3.70 \pm 0.20(n=6)$ \\
\hline
\end{tabular}
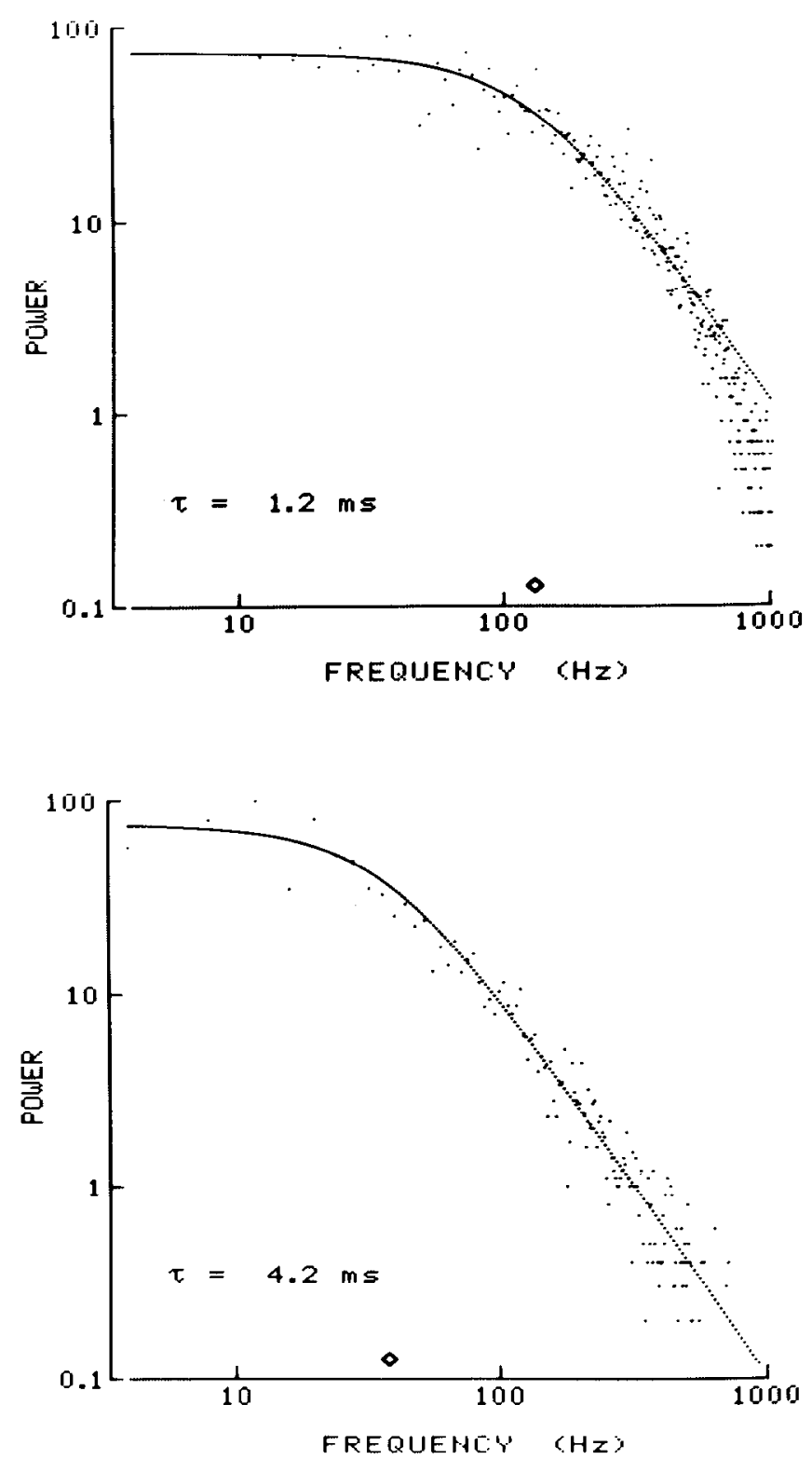

Figure 1. Power spectra of ACh-induced current fluctuations recorded from an innervated day-18 soleus endplate (top) and a day-18 endplate that had been denervated at day 10 (bottom). The two muscles were obtained from littermates. In each case, a simple Lorentzian curve fitted by least-squares regression is superimposed on the experimental spectrum. The diamonds indicate the half-power frequencies from which the apparent channel open times were derived. The rolloff of the upper experimental spectrum above $600 \mathrm{~Hz}$ was due to the limited frequency response of the recording electrode; this limitation was taken into account in fitting the Lorentzian curve. Temperature $=23^{\circ} \mathrm{C}$.

Tau at perijunctional sites. In most animals with denervated soleus muscles, we also measured $\tau$ in the contralateral innervated muscles. Tau at these innervated endplates decreased with age just as in normal animals (cf. Table II).

Unexpectedly, we found higher than normal levels of extrajunctional ACh sensitivity in many, but not all, of these contralateral innervated muscles. We could record ACh-induced membrane current fluctuations at sites up to several hundred micrometers away from the endplate. One explanation is that cutting the left sciatic nerve may have decreased the use of muscles in both limbs, thus slowing the loss of extrajunctional sensitivity (cf. Diamond and Miledi, 1962; Lomo and Rosenthal, 1972), but we have no evidence for this. Similar denerva- 
TABLE II

$\tau$ at developing innervated and denervated endplates

Tau was measured at 101 endplates in 21 innervated soleus muscles and at 28 endplates in 7 denervated soleus muscles. Some of the innervated muscles were contralateral to denervated muscles; others were from normal controls. Since the data from the two innervated groups were indistinguishable, the results were pooled. Endplates in innervated muscles, were located by focal extracellular recordings of spontaneous miniature endplate currents (Fischbach and Schuetze, 1980; Schuetze, 1980). Denervated endplates were identified by staining the muscle for extracellular AChE activity (see the text). At many of the innervated endplates, the power spectra of $\mathrm{ACh}$-induced current fluctuations were clearly biphasic, indicating that these endplates contained a mixture of channels with long and short apparent open times (Sakmann and Brenner, 1978; Fischbach and Schuetze, 1980). To get a rough estimate of the mean $\tau$ in these endplates, an apparent $\tau$ was obtained by fitting a simple Lorentzian curve to each spectrum (see "Materials and Methods"). The apparent $\tau$ values were averaged with the other $\tau$ estimates in the same age category. Biphasic spectra were also recorded from two endplates in the day- 18 muscle that was denervated at day 10 (see the text). The data labeled embryonic were obtained from embryonic rat leg muscle grown in dissociated cell culture (Fischbach and Schuetze, 1980); similar values have been found at newborn rat endplates in vivo (Sakmann and Brenner, 1978; Fisch bach and Schuetze, 1980). All data were normalized to $21^{\circ} \mathrm{C}$.

\begin{tabular}{|c|c|c|}
\hline \multirow{2}{*}{ Age } & \multicolumn{2}{|c|}{$\tau$} \\
\hline & Innervated & Denervated. \\
\hline days & \multicolumn{2}{|c|}{ mean $\pm S E M$, msec } \\
\hline Embryonic & $4.44 \pm 0.11(n=16)$ & \\
\hline $8-10$ & $2.70 \pm 0.21(n=18)$ & $4.98 \pm 0.43(n=3)$ \\
\hline $11-14$ & $2.30 \pm 0.12(n=42)$ & $4.49 \pm 0.19(n=16)$ \\
\hline $15-18$ & $1.89 \pm 0.10(n=41)$ & $3.92 \pm 0.27(n=9)$ \\
\hline
\end{tabular}

tion effects on innervated muscles have been reported in cats (Steinbach, 1981).

Whatever the cause, this observation allowed us to compare the apparent open time of AChRs in junctional and extrajunctional regions of the same, innervated fibers. Paired recordings were made from 44 fibers in 11 different muscles. In each case, we measured $\tau$ both at the endplate and at an extrajunctional site $200 \mu \mathrm{m}$ distant (about 10 to 15 endplate diameters) on the same fiber. The $\tau$ values measured at the two sites were in close agreement in all 44 fibers (Fig. 2). It is especially significant that brief open times were recorded at extrajunctional sites in many of these cases (Fig. 3). Thus, channel conversion is not restricted to the endplate membrane in rat soleus muscles.

Muscle ultrastructure during the $\tau$ decrease. One mechanism proposed to explain the $\tau$ decrease is that the nerve might induce a modification in the structure of endplate membrane (Brenner and Sakmann, 1983). In particular, it has been suggested that the appearance of postsynaptic folds, which is roughly coincident with channel conversion (Korneliussen and Jansen, 1976; Rosenthal and Taraskevich, 1977), may be related to the $\tau$ decrease (Fischbach and Schuetze, 1980; Michler and Sakmann, 1980; Brenner and Sakmann, 1983).

Our finding that channel conversion is not restricted to the endplate membrane makes this hypothesis unlikely. However, one could argue that in these experimental animals, denervation of one soleus muscle somehow induced structural changes, such as membrane folds, in the extrajunctional membrane of contralateral, innervated muscle fibers.

To test this explicitly, we selected an innervated fiber that had extrajunctional AChRs with short $\tau$ times and labeled the cell with an electron-dense marker using the Lucifer YellowDAB technique (Maranto, 1982). After preparing the tissue for electron microscopy, we examined the exact same extrajunctional region that had been assayed for apparent channel open time (see "Materials and Methods").
Although the tissue preservation after labeling was imperfect, the marked region was otherwise indistinguishable from extrajunctional regions of normal fibers (Fig. 4). Two other fibers were labeled and examined in the same way. In all cases, we could find no evidence for an ultrastructural correlate to the decrease in $\tau$. We conclude that the formation of postsynaptic folds is not directly related to channel conversion. Of course, there may be other changes in membrane structure, not apparent in our electron micrographs, that mediate channel conversion.

\section{Discussion}

These results show that neonatal denervation blocks the normal postnatal appearance of fast channel gating at rat endplates. Only long gating times were observed in endplates that were denervated prior to the onset of channel conversion. This is in contrast to results obtained in denervated adult muscles. When mature endplates are denervated, AChRs with short $\tau$ times can be found for up to at least several weeks later (Brenner and Sakmann, 1983). Thus, innervation appears to be required only for the initial appearance of fast gating at endplates and not for its maintenance.

One could argue that our inability to find channels with short $\tau$ times at the denervated endplates was an artifact of the AChE stain. This seems unlikely, since the AChE stain had no significant effect on $\tau$ measurements at either extrajunctional sites in denervated muscles or at endplates in innervated muscle fibers (Table I). Another possibility is that channel conversion did, in fact, proceed normally at the denervated endplates but that our recordings were dominated by contributions from slow channels in nearby perijunctional regions. This, too, seems unlikely. Focal extracellular recording has very good spatial resolution and assays essentially only those AChRs directly beneath the tip of the pipette (see Plate 1 in Schuetze, 1980). In two cases we did find evidence for a subpopulation of AChRs with short $\tau$ times at denervated endplates, but these endplates had been denervated at day 10, or 2 days after the normal onset of channel conversion (Sakmann and Brenner, 1978). Since we found no evidence for a significant subpopulation of channels with short $\tau$ times in other preparations, the simplest explanation is that the fast channels found in these two endplates had been present at the time of denervation.

It may be that denervation does not arrest channel conversion completely but merely delays it. We could not rule out this possibility because at long times after denervation we were unable to measure $\tau$, largely because of the accumulation of fat and connective tissue in the atrophic muscles. It appeared that neonatal denervation led to especially severe, rapid muscle atrophy. In one experiment we cut the sciatic nerve less than $24 \mathrm{hr}$ after birth. At postnatal day 18 the soleus muscle was so thin that we had trouble recognizing it during the dissection. Presumably, denervation during a period in which muscle growth is otherwise very rapid accelerates the atrophy.

In principle, denervalion might interfere with channel conversion either by decreasing muscle activity or by removing some other neuronal signal. A recent study that appeared after this work was completed suggests that activity is the key factor (Brenner et al., 1983). Channel conversion at developing ectopic endplates in adult rats continues after denervation in muscles that are given chronic, electrical stimulation. It is not known if denervation blocks channel conversion at ectopic endplates in inactive muscles, although our results would predict so.

The decrease in $\tau$ in rat muscle fibers, as in Xenopus (Kullberg et al., 1981), is not restricted to the endplate but also occurs in extrajunctional regions. It is not clear how far from the endplate channel conversion can occur: Our results show clearly that $\tau$ decreases for at least $200 \mu \mathrm{m}$ on either side of the endplate. In these muscles, $200 \mu \mathrm{m}$ is about 10 to 15 endplate diameters. Thus, channel conversion is not limited to the 


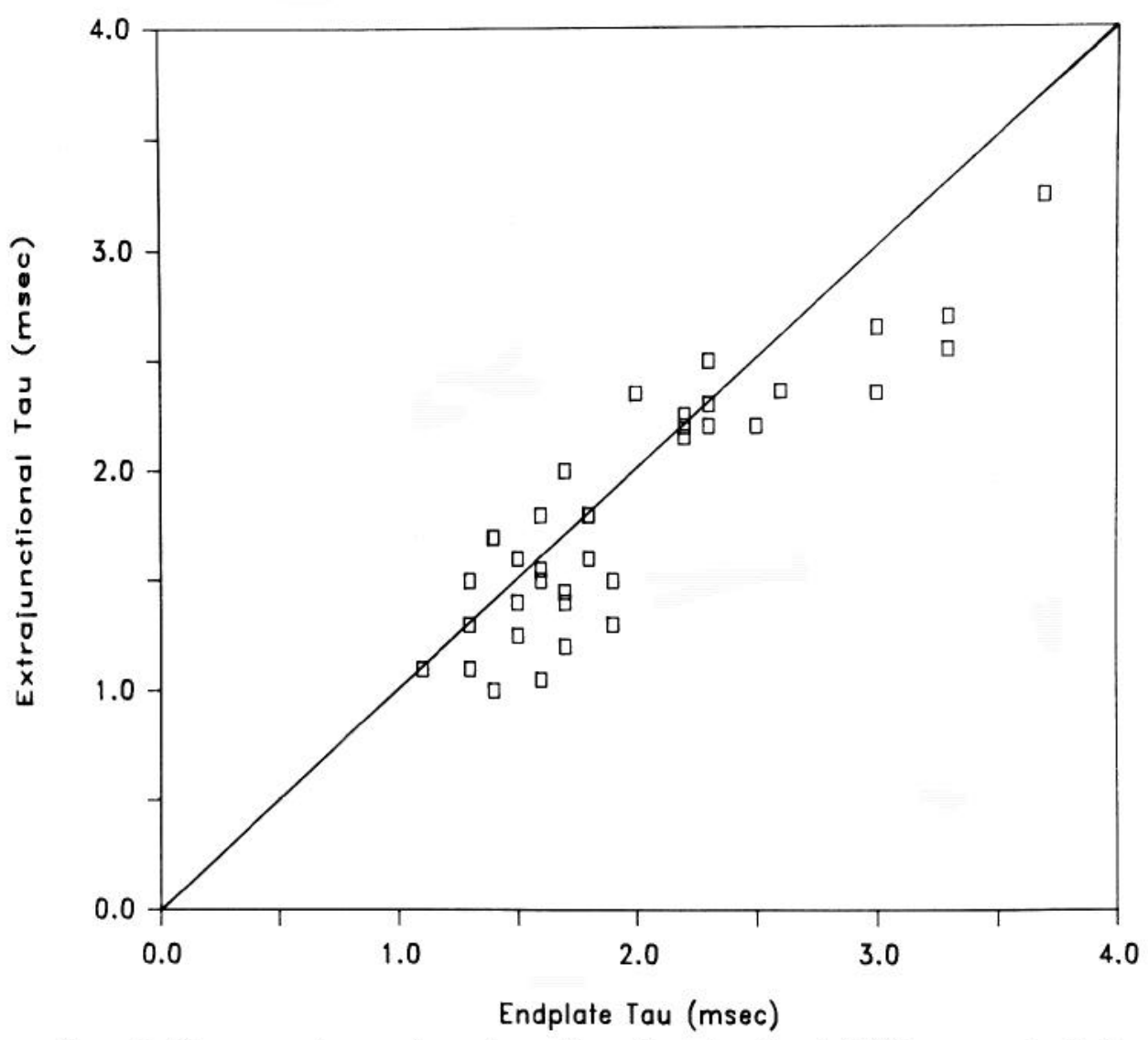

Figure 2. The apparent mean channel open time of extrajunctional AChRs compared with the apparent mean open time of junctional AChRs on the same fiber. Each symbol represents a different fiber in a day- 8 to day- 18 soleus muscle from an animal in which the contralateral soleus muscle was denervated between days 2 and 6 . Each extrajunctional site was approximately 200 $\mu \mathrm{m}$ distant from the endplate on the same fiber. The line has a slope of 1 and was not fitted to the data.

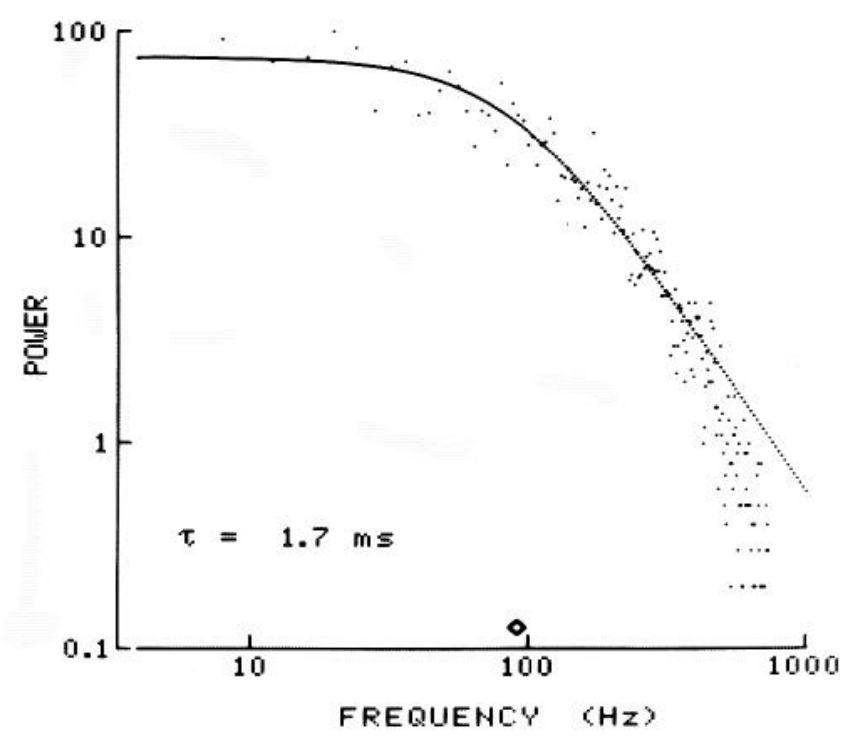

Figure 3. Spectrum obtained from an extrajunctional site $200 \mu \mathrm{m}$ distant from the endplate on the same fiber. The muscle was obtained from a day-10 rat in which the contralateral sciatic nerve had been cut at day 2 . Temperature $=22^{\circ} \mathrm{C}$.

immediate vicinity of the terminals but extends to regions that completely lack any postsynaptic specializations (Fig. 4). At sites still further from the endplate the ACh sensitivity was too low for accurate $\tau$ measurements. However, other evidence

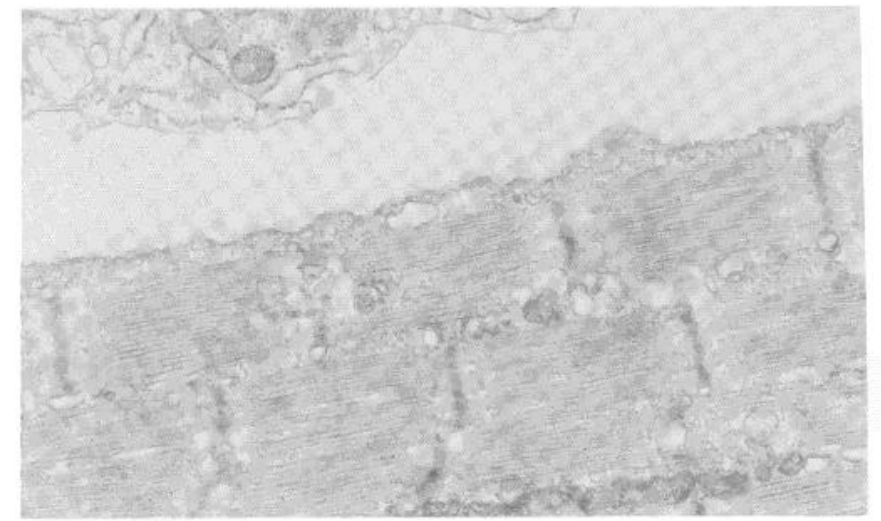

Figure 4. Electron micrograph of an extrajunctional site $200 \mu \mathrm{m}$ from the endplate of a day-10 innervated muscle. The contralateral sciatic nerve had been cut at day 3 . Tau was measured at precisely this site with fluctuation analysis and found to be $1.4 \mathrm{msec}$. Immediately afterward, the same site was labeled with an electron-dense marker and prepared for electron microscopy (see "Materials and Methods"). The somewhat blown-out appearance of this fiber is an artifact of the labeling procedure. Magnification $\times 11,500$.

indicates that channel conversion does not occur over the entire length of the fiber. In human intercostal muscle, $\tau$ is short at the endplate but long at the myotendinous junction (CullCandy et al., 1982). The sphere of influence of the nerve on channel gating remains to be determined.

Taken together, the above results suggest that channel gating 
is regulated differently at endplate and extrajunctional sites. It appears that the nerve is required only to initiate the $\tau$ decrease at endplates. Once channel conversion is complete, endplates contain fast channels independent of both activity and innervation. On the other hand, the gating kinetics of $A C h R s$ in extrajunctional regions apparently depends on innervation at all stages of maturity. When they are present in sufficiently mature, normally innervated muscles, extrajunctional AChRs have short apparent open times. Otherwise, $\tau$ at extrasynaptic sites is long.

The mechanism underlying channel conversion remains obscure. It is unlikely that the process requires replacing the entire receptor channel complex, for the AChR turnover rate is too slow to account for the more rapid appearance of fast channels (Michler and Sakmann, 1980; Reiness and Weinberg, 1981; but see Stanlcy and Drachman, 1983). Therefore, it seems more likely that channel conversion is mediated by modifying either the channel itself or its membrane environment. At present both possibilities remain equally likely. However, the finding that channel conversion occurs extrajunctionally indicates that the mechanism is independent of the formation of postsynaptic folds and of other developmental events restricted to the endplate region.

\section{References}

Anderson, C. R., and C. F. Stevens (1973) Voltage clamp analysis of acetylcholine produced endplate current fluctuations at frog neuromuscular junction. J. Physiol. (Lond.) 235: 655-691.

Anderson, M. J., M. W. Cohen, and E. Zorychta (1977) Effects of innervation on the distribution of acetylcholine receptors on cultured muscle cells. J. Physiol. (Lond.) 268: 731-756.

Berg, D. K., and Z. W. Hall (1975) Loss of $\alpha$-bungarotoxin from junctional and extrajunctional acetylcholine receptors in rat diaphragm muscle in vivo and in organ culture. J. Physiol. (Lond.) 252 $771-789$.

Brenner, H. R., and B. Sakmann (1983) Neurotrophic control of channel properties at neuromuscular synapses of rat muscle. J. Physiol. (Lond.) 337: 159-171.

Brenner, H. R., Th. Meier, and B. Widmer (1983) Early action of nerve determines motor endplate differentiation in rat muscle. Nature 305 : $536-537$.

Cull-Candy, S. G., R. Miledi, and O. D. Uchitel (1982) Properties of junctional and extrajunctional acetylcholine-receptor channels in organ cultured human muscle fibres. J. Physiol. (Lond.) 333: 251267.

Dennis, M. J. (1981) Development of the neuromuscular junction: Inductive interactions between cells. Annu. Rev. Neurosci. 4: 43-68.

Diamond, J., and R. Miledi (1962) A study of foetal and new-born rat muscle fibers. J. Physiol. (Lond.) 162: 393-408.

Fambrough, D. M. (1979) Control of acetylcholine receptors in skeletal muscle. Physiol. Rev. 59: 165-227.
Fischbach, G. D., and S. M. Schuetze (1980) A postnatal decrease in acetylcholine channel open time at rat endplates. J. Physiol. (Lond.) 303: 125-137.

Frank, E., and G. D. Fischbach (1979) Early events in neuromuscular junction formation in vitro: Induction of acetylcholine receptor clusters in the postsynpatic membrane and morphology of newly formed nerve-muscle synapses. J. Cell Biol. 83: 143-158.

Hamill, O. P., A. Marty, E. Neher, B. Sakmann, and F. J. Sigworth (1981) Improved patch-clamp techniques for high-resolution current recording from cells and cell-free membrane patches. Pflügers Arch 391: 85-100.

Karnovsky, M. L., and L. Roots (1964) A "direct-coloring" thiocholine method for cholinesterases. J. Histochem. Cytochem. 12: 219-221.

Katz, B., and R. Miledi (1972) The statistical nature of the acetylcholine potential and its molecular components. J. Physiol. (Lond.) 224. $665-699$.

Korneliussen, H., and J. K. S. Jansen (1976) Morphological aspects of the elimination of polyneuronal innervation of skeletal muscle of newborn rats. J. Neurocytol. 5: 591-604.

Kullberg, R. W., P. Brehm, and J. H. Steinbach (1981) Nonjunctional acetylcholine receptor channel open time decreases during development of Xenopus muscle. Nature 289: 411-413.

Lomo, T., and J. Rosenthal (1972) Control of ACh sensitivity by muscle activity in the rat. J. Physiol. (Lond.) 221: 493-513.

Maranto, A. R. (1982) Neuronal mapping: A photooxidation reaction makes Lucifer Yellow useful for electron microscopy. Science 217: 953-955.

Michler, A., and B. Sakmann (1980) Receptor stability and channel conversion in the subsynaptic membrane of the developing mammalian neuromuscular junction. Dev. Biol. 80: 1-17.

Miledi, R., and C. R. Slater (1968) Electrophysiology and electronmicroscopy of rat neuromuscular junctions after nerve degeneration. Proc. R. Soc. Lond. (Biol.) 169: 289-306.

Reiness, C. G., and C. B. Weinberg (1981) Metabolic stabilization of acetylcholine receptors at newly formed neuromuscular junctions in the rat. Dev. Biol. 84: 247-254.

Rosenthal, J. L., and P.S. Taraskevich (1977) Reduction of multiaxonal innervation at the neuromuscular junction of the rat during development. J. Physiol. (Lond.) 270: 299-310.

Sakmann, B. (1978) Acetylcholine-induced ionic channels in rat skeletal muscle. Fed. Proc. 37: 2654-2659.

Sakmann, B., and H. R. Brenner (1978) Change in synaptic channel gating during neuromuscular development. Nature 276: 401-402.

Schuetze, S. M. (1980) The acetylcholine channel open time in chick muscle is not decreased following innervation. J. Physiol. (Lond.) 303: 111-124.

Schuetze, S. M., and G. D. Fischbach (1978) Channel open time decreases postnatally in rat synaptic acetylcholine receptors. Soc. Neurosci. Abstr. 4: 374

Stanley, E. F., and D. B. Drachman (1983) Rapid degradation of "new" acetylcholine receptors at neuromuscular junctions. Science 222: $67-$ 69.

Steinbach, J. H. (1981) Neuromuscular junctions and $\alpha$-bungarotoxinbinding sites in denervated and contralateral cat skeletal muscles. J. Physiol. (Lond.) 313: 513-528. 